\title{
Meiotic breakpoint mapping of a proposed X linked visual loss susceptibility locus in Leber's hereditary optic neuropathy
}

\author{
Herlina Y Handoko, Pratyaksha J Wirapati, Herawati A Sudoyo, Mulia Sitepu, \\ Sangkot Marzuki
}

\begin{abstract}
Leber's hereditary optic neuropathy (LHON) is a maternally inherited degenerative disorder characterised by an acute or subacute optic nerve degeneration resulting in visual failure. Mitochondrial DNA mutations have been reported and a nuclear modifier gene(s) on the $X$ chromosome is thought to play an important role in the onset of this disorder. We analysed a LHON family with a novel and more accurate approach using $27 \mathrm{X}$ chromosomal microsatellite markers. Meiotic breakpoint mapping and two point lod score did not point to any particular area on the $X$ chromosome which might contain the $X$ susceptibility locus.

(F Med Genet 1998;35:668-671)
\end{abstract}

Keywords: Leber's hereditary optic neuropathy (LHON); mitochondrial DNA; X linked susceptibility locus; linkage

A range of missense mutations in the mitochondrial DNA (mtDNA) has now been shown to be associated with Leber's hereditary optic neuropathy (LHON). ${ }^{2}$ However, there remain several confusing aspects of its inheritance, which cannot be explained by mitochondrial inheritance alone. These include male predominance, unaffected females transmitting the disease, and later age of onset for females.

The most compelling explanation for complex inheritance is the existence of an interaction between an $\mathrm{X}$ linked nuclear modifier gene and the mtDNA defect. Segregation analyses ${ }^{3-4}$ have confirmed this possibility, taking into account the $\mathrm{X}$ chromosome inactivation in heterozygous females. However, a study on three Tasmanian families using $15 \mathrm{X}$ chromosome markers ${ }^{6}$ suggested exclusion of the $\mathrm{X}$ chromosome. Suggestion of linkage with $\mathrm{DXS}^{7}$ was later re-evaluated by Juvonen $e t a l^{8}$ as no evidence for linkage, as also suggested also by other investigators. ${ }^{9}{ }^{10}$ Results against the presence of an $\mathrm{X}$ linked susceptibility locus based on $\mathrm{X}$ inactivation analysis were reported by several investigators. ${ }^{112}$

These reports prompted us to evaluate a subset of a large, four generation LHON family, ${ }^{13}$ using a meiotic breakpoint mapping approach with many more markers along the $\mathrm{X}$ chromosome and tighter criteria in choosing the members of the family for evaluation. We limited our study to members of one family to avoid the genetic heterogeneity which might exist between families. Ophthalmological investigations have excluded other causes of impaired vision in this family. All relevant family members were homoplasmic for the 11778 G>A mtDNA mutation, which allowed us to concentrate on the $\mathrm{X}$ chromosome without worrying about mtDNA heteroplasmicity. The fourth generation was not included in this study because of the late onset of expression of the disease. The youngest unaffected male in the third generation was 26 years old (J329), while the average age of onset was 19 years old.

The most important feature of the present study is the approach taken in choosing a critical subset of the family (fig 1 ) which focuses on the female members of the second generation, but only on the male members of the third generation. The unaffected males showed no subclinical or insidious course on ophthalmological investigation. This approach allowed us to follow only three $\mathrm{X}$ chromosomes from the grandparents (that is, two from the grandmother J101 and one from the grandfather J102) and to determine their segregation and meiotic breakpoint in the grandsons. Since all the grandsons are homoplasmic for the 11778 mutation and carry only one copy of the $\mathrm{X}$ chromosome, the shared chromosomal segments among the affected subjects should contain the putative $\mathrm{X}$ linked susceptibility locus. This approach also eliminates the need to use parameters such as the penetrance of $L H O N$ in heterozygote females, and because we made fewer assumptions, we avoided the mistake of rejecting a correct model.

The $\mathrm{X}$ chromosomes of the living members in this family subset were analysed using 27 polymorphic markers located about $10 \mathrm{cM}$ apart. Three regions of the $\mathrm{X}$ chromosomes were saturated with more markers, as these areas may contain candidate loci: (1) around DXS7 as an area reported ${ }^{7}$ to show evidence of linkage, (2) around the ANT2 gene (coding for a mitochondrial ADP/ATP translocase) tightly linked to DXS425, ${ }^{14}$ and (3) around NDUFA1 (coding for a subunit of $\mathrm{NADH}$ :ubiquinone oxidoreductase) which has been mapped to $\mathrm{Xq} 24 .^{15}$

We had no DNA from the first generation (J101 or J102), but could deduce their genotypes from the second generation (daughters $\mathrm{J} 202$, J204, J205, J207 and son J208). As a male, $\mathrm{J} 208$ (fig 1) could have received the $\mathrm{X}$ chromosome only from his mother (J101), and thus we can determine one of J101 alleles, some with 


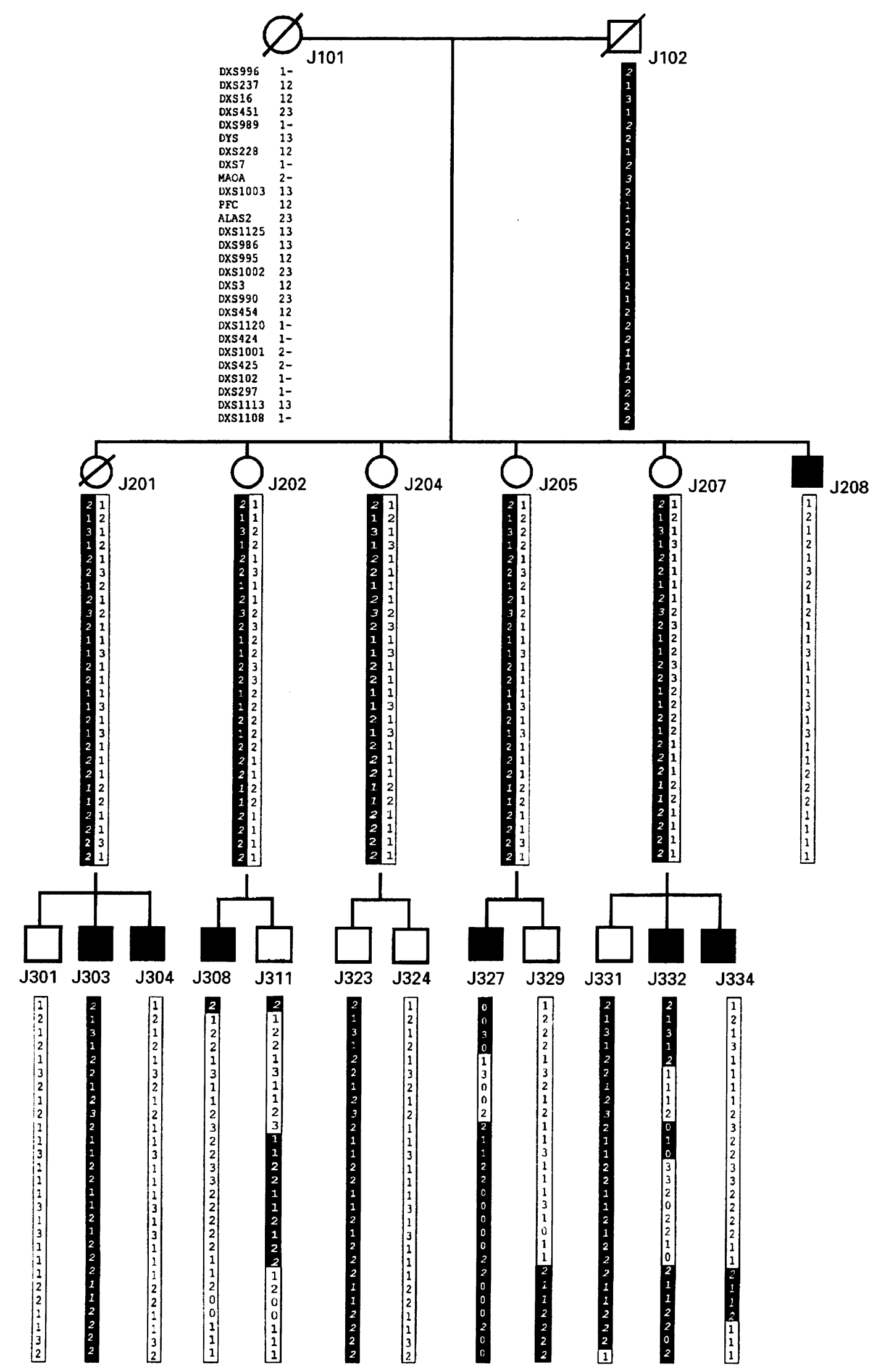

Figure 1 A subset of the LHON family included in the meiotic breakpoint and two point linkage analyses. Using this subset, only three $X$ chromosomes segregate from the grandparents to the grandsons, making it possible to follow the meiotic breakpoints and to look for shared chromosomal segments among the affected subjects. Black boxes represent the haplotype of F102 predicted from the genotypes of his daughters and son (bold numbers represent genotypes determined with certainty, italic numbers represent most probable genotypes). In the daughters'gametes the $X$ chromosomes from 1102 might recombine with the ones from $\mathcal{Y 1} 101$ which can be observed in the grandsons' $X$ chromosomes. Microsatellite markers used are printed on the upper left hand side of the pedigree. Zero $(0)$ means genotype was not available. Six more markers (DXS1060, DXS987, DXS1226, DXS1202, DXS1214, and DXS1055) have been used to analyse this family without any changes to the overall result. 
Table 1 Two point $Z$ values for linkage between a putative nuclear modifying locus for developing LHON and $27 X$ chromosomal marker loci

\begin{tabular}{|c|c|c|c|c|c|c|c|c|c|}
\hline Locus & $\theta=0$ & $\theta=0.05$ & $\theta=0.1$ & $\theta=0.15$ & $\theta=0.2$ & $\theta=0.25$ & $\theta=0.3$ & $\theta=0.35$ & $\theta=0.4$ \\
\hline DXS996 & -0.96276 & -0.78677 & -0.64144 & -0.51747 & -0.40999 & -0.3159 & -0.23307 & -0.16022 & -0.09685 \\
\hline DXS237 & -0.74217 & -0.75043 & -0.69653 & -0.58933 & -0.46154 & -0.33924 & -0.23413 & -0.14904 & -0.08299 \\
\hline DXS16 & -1.72556 & -1.1284 & -0.89176 & -0.70496 & -0.53781 & -0.3892 & -0.26175 & -0.15764 & -0.07844 \\
\hline DXS451 & -1.72556 & -1.1631 & -0.82861 & -0.57008 & -0.37438 & -0.23167 & -0.13183 & -0.06578 & -0.02577 \\
\hline DXS989 & -1.2608 & -1.04273 & -0.75052 & -0.5091 & -0.32785 & -0.1954 & -0.1015 & -0.039 & -0.00316 \\
\hline DYS & -1.72556 & -0.53559 & -0.12647 & 0.08068 & 0.1841 & 0.22261 & 0.21724 & 0.18216 & 0.1285 \\
\hline DXS228 & -0.53371 & -0.51935 & -0.47061 & -0.40046 & -0.32215 & -0.24515 & -0.17497 & -0.11441 & -0.06474 \\
\hline DXS7 & -1.17466 & -0.55806 & -0.3004 & -0.15234 & -0.06076 & -0.00447 & 0.02715 & 0.04018 & 0.0384 \\
\hline MAOA & -1.26068 & -0.36574 & -0.08686 & 0.05655 & 0.13207 & 0.16479 & 0.16724 & 0.14693 & 0.10921 \\
\hline DXS1003 & -1.72556 & -1.43163 & -1.04802 & -0.75128 & -0.52229 & -0.34455 & -0.209 & -0.11014 & -0.0442 \\
\hline PFC & -1.72556 & -0.65399 & -0.40036 & -0.24878 & -0.1417 & -0.06435 & -0.01149 & 0.01953 & 0.03062 \\
\hline ALAS2 & -1.72556 & -0.99486 & -0.6386 & -0.40801 & -0.24527 & -0.12869 & -0.04817 & 0.00182 & 0.02482 \\
\hline DXS1125 & -1.72556 & -1.26919 & -0.8136 & -0.51546 & -0.30944 & -0.16384 & -0.06372 & -0.0013 & 0.02801 \\
\hline DXS986 & -1.72556 & -1.34317 & -0.92269 & -0.63369 & -0.42536 & -0.26971 & -0.15374 & -0.07126 & -0.01916 \\
\hline DXS995 & 0.13271 & 0.10979 & 0.10707 & 0.115 & 0.12435 & 0.12856 & 0.12383 & 0.10845 & 0.08211 \\
\hline DXS1002 & -1.72557 & -0.9981 & -0.64207 & -0.41024 & -0.24594 & -0.12793 & -0.04631 & 0.00432 & 0.02736 \\
\hline DXS3 & -1.09878 & -0.96961 & -0.78102 & -0.60335 & -0.45227 & -0.32682 & -0.22368 & -0.14037 & -0.07552 \\
\hline DXS990 & -1.72557 & -1.06869 & -0.75966 & -0.53544 & -0.36003 & -0.22315 & -0.1202 & -0.04826 & -0.00525 \\
\hline DXS454 & -0.74216 & -0.5997 & -0.40251 & -0.23659 & -0.11653 & -0.03661 & 0.01136 & 0.03441 & 0.03793 \\
\hline DXS 1120 & -1.04132 & -0.83952 & -0.61657 & -0.42685 & -0.27907 & -0.16845 & -0.08886 & -0.03533 & -0.00423 \\
\hline DXS424 & -0.96276 & -0.79106 & -0.65246 & -0.53655 & -0.43604 & -0.34582 & -0.26281 & -0.18583 & -0.11527 \\
\hline DXS1001 & -1.04132 & -0.87793 & -0.72737 & -0.58598 & -0.456 & -0.34018 & -0.24013 & -0.15624 & -0.08838 \\
\hline DXS425 & -0.93662 & -0.77656 & -0.64293 & -0.52469 & -0.41835 & -0.3229 & -0.238 & -0.16344 & -0.09902 \\
\hline DXS102 & -0.4158 & -0.2773 & -0.20215 & -0.16085 & -0.13853 & -0.12538 & -0.11391 & -0.09832 & -0.07472 \\
\hline DXS297 & -1.13729 & -0.98132 & -0.80819 & -0.62937 & -0.46361 & -0.32168 & -0.2068 & -0.1186 & -0.05563 \\
\hline DXS1113 & -1.72556 & -0.6738 & -0.37935 & -0.20591 & -0.09028 & -0.01293 & 0.03449 & 0.05651 & 0.05627 \\
\hline DXS1108 & -0.77435 & -0.6681 & -0.54875 & -0.43185 & -0.93875 & -0.95497 & -0.16181 & -0.10179 & -0.05534 \\
\hline
\end{tabular}

certainty, some with high probability. For instance, for DXS996, one of J101's alleles must be a 1 which she passed on to her son J208. The genotypes of J202, J204, J205, and J207 are all 12 which leads to two possibilities: (1) the genotype of J101 is 12 and J102 is 1 or (2) the genotype of J101 is $1-$ (- denotes any genotype) and that of J102 is 2 . Because all daughters are 12 , the latter is more likely (J102 passes on his allele to every daughter, while J101 has only a $50 \%$ chance of passing on each of her two alleles). The genotypes of J101 and J102 can be deduced with certainty for marker DXS237. One of the alleles of J101 must be a 2 which is passed to J208. Her second allele must be a 1 and J102 must also be a 1 , so that $\mathrm{J} 202$ can inherit 11 from her parents.

The haplotype of $\mathrm{J} 102$ thus predicted (in black in fig 1) would be passed on to each daughter intact (because there is no recombination on the $\mathrm{X}$ chromosome in male gametes, except in the pseudoautosomal regions which were not included in this study). On the other hand, in the females of the second generation the $\mathrm{X}$ chromosomes could cross over giving recombinant chromosomes in the grandsons (a black and white pattern in the third generation of fig 1). The parts of the chromosomes shared by two subjects can be deduced easily from the recombination pattern.

No particular candidate region was found to be shared among affected males in the third generation (fig 1). Most significantly, an identical haplotype was shared by $\mathrm{J} 303$ (affected) and J323 (unaffected) on the basis of the 27 markers analysed. If, because of the possibility of later age of onset, all the males in the third generation become affected later on, we still cannot find any particular segment shared by each one of the males in the third generation.

Two point lod scores (table 1) were calculated with parameters similar to those of Chalmers et al, ${ }^{11}$ but the female phenotype was considered unknown (thus the penetrance in females does not have to be assumed), and a phenocopy rate of $10 \%$ was assumed. We consider the nuclear factor as a susceptibility factor, not a necessary one.

With the parameters we applied above, the lod scores ranged between -1.726 and 0.223 and thus the possibility of the presence of a nuclear modifier on the $\mathrm{X}$ chromosome cannot be ruled out totally. Multipoint analysis did not change the overall results of the two point analysis. The lod scores lower than -2 were obtained when phenocopies were not taken into account. Our results suggest that the nuclear modifier gene, if it is located on the $\mathrm{X}$ chromosome, is not inherited in a simple, straightforward manner as in major gene inheritance. This nuclear modifier could work as a susceptibility factor, increasing the risk of a person with the mtDNA mutation being affected. We will need to examine a larger number of affected subjects using a model free approach to be able to detect this nuclear modifier gene(s).

We thank Professor Grant Sutherland and Dr John Mulley who allowed us to carry out the linkage analysis in their laboratories allowed us to carry out the linkage analysis in their laboratories at the Women's and Children's Hospital, Adelaide, Australia. HYH thanks the Human Genome Organization for the HUGO Travel Award. This work was supported by the PEGELKOP
grants from PT INTI and Krakatau Steel through the Agency grants from PT INTI and Krakatau Steel throu
for Strategic Industries, Republic of Indonesia.

1 Wallace DC, Singh G, Lott MT, et al. Mitochondrial DNA Wallace DC, Singh G, Lott MT, et al. Mitochondrial DNA ropathy. Science 1988;242:1427-30.

2 Wallace DC. Diseases of the mitochondrial DNA. Annu Rev Biochem 1992;61:1175-212.

$3 \mathrm{Bu} \mathrm{X}$, Rotter Л. X chromosome-linked and mitochondrial gene control of Leber hereditary optic neuropathy: evidence from segregation analysis for dependence on $\mathrm{X}$ chromosome inactivation. Proc Natl Acad Sci USA 1991;88:8198-202.

4 Nakamura $M$, Fujiwara Y, Yamamoto $M$. The two locus control of Leber hereditary optic neuropathy and a high penetrance in Japanese pedigrees. Hum Genet 1993;91:339

5 Harding AE, Sweeney MG, Govan GG, et al. Pedigree analysis in Leber hereditary optic neuropathy families with a pathogenic mtDNA mutation. Am $\mathcal{F}$ Hum Genet 1995;57: 77-86.

6 Chen JD, Cox I, Denton MJ. Preliminary exclusion of an $\mathrm{X}$-linked gene in Leber optic atrophy by linkage analysis. Hum Genet 1989:82:203-7.

7 Vilkki J, Ott J, Savontaus ML, et al. Optic atrophy in Leber hereditary optic neuroretinopathy is probably determined by an X-chromosomal gene closely linked to DXS7. Am $\mathcal{F}$ Hum Genet 1991;48:486-91. 
8 Juvonen V, Vilkki J, Aula P, et al. Reevaluation of the linkage of an optic atrophy susceptibility gene to X-chromosomal roretinopathy (LHON). Am f Hum Genet 1993;53:289-92.

9 Carvalho MRS, Muller B, Rotzer E, et al. Leber's hereditary optic neuroretinopathy and the X-chromosomal susceptibility factor: no linkage to DXS7. Hum Hered 1992;42:31620 .

10 Sweeney MG, Davis MB, Lashwood A, et al. Evidence against an X-linked locus close to DXS7 determining against an X-linked locus close to DXS7 determining visual loss susceptibility in British and Italian families with 51:741-8.

11 Chalmers RM, Davis MB, Sweeney MG, et al. Evidence against an X-linked visual loss susceptibility locus in Leber hereditary optic neuropathy. Am ff Hum Genet 1996;59: 103-8.
12 Oostra RJ, Kemp S, Bolhuis PA, et al. No evidence for "skewed" inactivation of the X-chromosome as cause of Leber's hereditary optic neuropathy in female carriers. Hum Genet 1996;97:500-5.

13 Sudoyo H, Sitepu M, Malik S, et al. Leber's hereditary optic neuropathy in Indonesia - two families with the mtDNA $11778 \mathrm{G}>\mathrm{A}$ and $14484 \mathrm{~T}>\mathrm{C}$ mutations. Hum Mutat 1998; suppl 1: $S 271-4$.

14 Schiebel K, Mertz A, Winkelmann M, et al. Localization of the adenine nucleotide translocase gene ANT2 to chromosome Xq24-q25 with tight linkage to DXS425. Genomics 1994;24:605-6.

15 Zhuchenko O, Wehnert $M$, Bailey J, et al. Isolation, mapping and genomic structure of an X-linked gene for a subunit of human mitochondrial complex I. Genomics 1996;37:281-8. 